\title{
A brief discussion on High-entropy alloys vs Compositionally Complex alloys
}

\author{
Mainak Saha* \\ Department of Metallurgical and Materials Engineering, National Institute of Technology, \\ Durgapur-713209, West Bengal, India \\ Department of Metallurgical and Materials Engineering, Indian Institute of Technology \\ Madras, Chennai-600036, Tamil Nadu, India
}

Email id: $\underline{\text { mm18d704@smail.iitm.ac.in }}$

ORCID: 0000-0001-8979-457x

\begin{abstract}
High-entropy alloys (HEAs) are of great interest in the field of materials science and engineering. Unlike conventional alloys, which contain a maximum of two base metals, this new category of materials consists of multi-principle elements and the possible number of alloy compositions, in case of HEAs, is outstandingly higher than that for conventional alloys. Here, a review on the high-entropy alloys, has been provided, on the issue whether the earliest definition of High- Entropy alloys, proposed in 2004, is consistent with one of the four-component equiatomic Compositionally complex alloys exhibiting a "High-entropy" effect.
\end{abstract}

Keywords: High-entropy alloys, compositional complexity, valence electron concentration, Miedema model, paired sigma forming element

\section{Introduction}

Since ancient times, human civilisation has always been striving towards the development of new classes of materials ${ }^{[1]}$, which have played a vital role for more than thousands of years. Since the Bronze age, alloys have been developed based on "base element paradigm" i.e. by using one or at most, 2 base elements, for example iron in steels or Ni in Ni-based superalloys. In 2004, a new paradigm of designing alloys, was proposed independently by Prof. J.W. Yeh and Prof. Brian Cantor. which eschewed the "base element" approach. Prof. J.W. Yeh named these materials as "High-entropy alloys" and there is already a highentropy alloy, popularly named as Cantor alloy, after its discoverer, which forms a single phase FCC solid solution at room temperature. By the term "Highentropy", it is clear that the configurational entropy of this alloy is high due to random mixing of the elements, in this case. The new theory has basically opened up new avenues in research of engineering materials. In conventional 'base metal' approach, only $n$ different alloys may be obtained with $n$ base metals. However, if a combination of $p$ elements are to be selected from a total of $n$ elements, to form an equiatomic alloy $(\mathrm{p}=2,3,4, \ldots .$.$) , the total number, \mathrm{N}$, of the alloys, may be increased from $n$ to $\mathrm{N}=2^{\mathrm{n}}-\mathrm{n}-1 .{ }^{[2]}$ So, from the mathematical expression, when 
$\mathrm{n}<3$, the two approach do not differ considerably. However, with increasing $\mathrm{n}$, the two approach differ largely, due to exponential dependence of $\mathrm{N}$ on $\mathrm{n}^{[16]}$.

2. The concept of HEAs ${ }^{[2][3][4][5][6][7][8][9][17]}$ 
Considering an ideal solution, the configurational entropy of mixing per mole, may be expressed as $\Delta_{\text {mix }} \mathrm{S}=-\mathrm{R} \Sigma \mathrm{C}_{\mathrm{i}} \ln \mathrm{C}_{\mathrm{i}}$, where $\mathrm{R}$ is universal gas constant and equal to $8.314 \mathrm{~J} / \mathrm{molK}, \mathrm{C}_{\mathrm{i}}$ is the mole fraction of ith element and $\mathrm{i}$ ranges from 1 to $\mathrm{n}$. The closer the alloy composition to the central region, the larger is the value of $\Delta_{\text {mix }} S_{\mathrm{m}}$ which reaches a maximum of $9.15 \mathrm{~J} / \mathrm{molK}$ at the centre. Consequently, the molar entropy of mixing $\left(\Delta_{\text {mix }} S_{m}\right)$, for an equiatomic HEA, is given by

$$
\Delta_{\text {mix }} \mathrm{S}=\mathrm{R} \ln \mathrm{n}
$$

With the "High-entropy" effect, the random solid solution may be stabilised against the intermetallic compounds when numerous elements are mixed in equimolar fraction. According to Zhang, et al. two additional parameters have been proposed for design of HEAs, namely $\delta$ (atomic size difference) ${ }^{[2]}$ and $\Delta_{\text {mix }} \mathrm{H}$ which are mathematically expressed as follows(the binary molar enthalpy of mixing, in case of binary system $\mathrm{i}-\mathrm{j}$, is given by $\Delta_{\text {mix }} \mathrm{H}_{\mathrm{ij}}{ }^{[3]}$, calculated according to the Miedema model):

$$
\delta=\sqrt{\sum_{i=1}^{n} c_{i}\left(1-\frac{r_{i}}{\bar{r}}\right)^{2}}
$$

$r_{i-}$ atomic radius of $i t h$ element

$C_{i-}$ atom fraction of $i t h$ element

$\bar{r}$ - average atomic radius $=\sum_{i=1}^{n} C_{i} r_{i}$

$$
\Delta_{\text {mix }} \mathrm{H}_{m}=\sum_{\mathrm{i}=1, \mathrm{i} \neq \mathrm{j}}^{\mathrm{N}} \Omega_{\mathrm{ij}} \mathrm{C}_{\mathrm{i}} \mathrm{C}_{\mathrm{j}}=\sum_{\mathrm{i}=1, \mathrm{i} \neq \mathrm{j}}^{\mathrm{N}} 4 \Delta_{m i x} \mathrm{H}_{\mathrm{ij}} \mathrm{C}_{\mathrm{i}} \mathrm{C}_{\mathrm{j}}
$$

$\Omega_{i j}$ - melt interaction parameter (Miedema ${ }^{[1]}$ model)

$\Delta_{\text {mix }} H_{\mathrm{m}}$ - Enthalpy of mixing. This is found to be $<0$ for compounds and $>0$ for random solid solutions, following the Quasi-Chemical theory ${ }^{[11]}$.

$C_{i}$-atom fraction of ith element

Atomic size mismatch parameter $(\boldsymbol{\delta})$ : This parameter quantifies atomic radius mismatch.

According to Yeh, et al. ${ }^{[2]}$ symbolically, for HEAs

$\mathrm{TS}_{\text {sys }} \gg>\max \left(\left|\mathrm{H}_{\mathrm{i}}\right|\right) \quad(\mathrm{i}=1,2, \ldots \ldots . \mathrm{m})$ 
where $S_{\text {sys }}$ is the configurational entropy of the alloy, $H_{i}$ is the enthalpy of formation of the ith phase, assuming $\mathrm{m}$ phases in total. However, in their early work, Yeh, et al. proposed a criterion $\Delta_{\text {mix }} S_{\mathrm{m}}>=1.6 \mathrm{R}$, corresponding to mixing of at least 5 elements in equiatomic composition.

Similarly, Zhang, et al. proposed another parameter, $\Omega,{ }^{[5]}$ showing the relative dominance of Enthalpy of mixing $\left(\Delta_{\text {mix }} H_{\mathrm{m}}\right)$ given as

$$
\Omega=\mathrm{T}_{\mathrm{m}} \Delta_{\text {mix }} \mathrm{S}_{\mathrm{m}} /\left|\Delta_{\text {mix }} \mathrm{H}_{\mathrm{m}}\right|
$$

Where, $\mathrm{T}_{\mathrm{m}^{-}}$absolute melting point of the alloy.

$\Omega<1:\left|\Delta_{\text {mix }} H_{m}\right|<\Delta_{\text {mix }} S_{m}$, implies that, intermetallic compound formation or segregation is favoured.

$\Omega>1:\left|\Delta_{\text {mix }} H_{m}\right|>\Delta_{\text {mix }} S_{m}$, implies that, random solid solution is favoured.

Valence electron concentration (VEC) ${ }^{[6]}$ : For electron compounds, VEC is used as a useful parameter in finding its formation propensity. This parameter is determined from the number of electrons in $4 \mathrm{~s}$ and $3 \mathrm{~d}$ orbitals of individual elements( in ground state)

$$
V E C=\sum_{i=1}^{n} C_{i}(V E C)_{i}
$$

$(\mathrm{VEC})_{i}$ valence electron concentration of element $i$.

Electronegativity $(\Delta \chi)^{[6]}$ :

$$
\Delta \chi=\sqrt{\sum_{i=1}^{N} C_{i}\left(\chi_{i}-\bar{\chi}\right)^{2}}
$$

$\chi_{\mathrm{i}}$ - electronegativity of $i t h$ lement.(according to Pauling Scale)

According to Hemphill et at. ${ }^{[7]}$ the total entropy of mixing $\mathrm{S}_{\mathrm{T}}$ per spherefor a hard sphere system with different sized particles, may be expressed as follows:

$$
\mathrm{S}_{\mathrm{T}}\left(\mathrm{c}_{\mathrm{i}}, \mathrm{r}_{\mathrm{i}}, \mathcal{E}\right)=\mathrm{S}_{\mathrm{C}}\left(\mathrm{c}_{\mathrm{i}}\right)+\mathrm{S}_{\mathrm{E}}\left(\mathrm{c}_{\mathrm{i}}, \mathrm{r}_{\mathrm{i}}, \mathcal{E}\right)
$$

Where,

$\mathrm{S}_{\mathrm{E}-}$ excess entropy of mixing

$\mathrm{S}_{\mathrm{C}}=-\mathrm{k} \Sigma \mathrm{C}_{\mathrm{i}} \ln \mathrm{C}_{\mathrm{i}}$

k- Boltzmann constant $=1.38^{*} 10^{\wedge}(--23) \mathrm{J} / \mathrm{K}$ 
$\mathrm{c}_{\mathrm{i}}, \mathrm{r}_{\mathrm{i}}, \boldsymbol{\varepsilon}$ - atomic concentration, atomic radius and overall packing density, respectively.

\section{$\Phi$-parameter ${ }^{[8]}$}

Ye, et al. assumed that $\mathrm{S}_{\mathrm{sys}} \sim \mathrm{S}_{\mathrm{T}} \sim \mathrm{S}_{\mathrm{C}}-\left|\mathrm{S}_{\mathrm{E}}\right|$ and accordingly proposed a parameter $\Phi$, expressed mathematically as:

$\Phi=\left(\mathrm{S}_{\mathrm{C}^{-}}\left|\Delta_{\text {mix }} \mathrm{H}_{\mathrm{m}}\right| / \mathrm{T}_{\mathrm{m}}\right) /\left|\mathrm{S}_{\mathrm{E}}\right|$

If $\Phi<\Phi_{C}$, HEAs form multiple phases and even an amorphous structure. And if $\Phi>\Phi_{\mathrm{C}}$, HEAs form single phase solid solution.

In the strictest sense, a solid solution phase is preferred to an inter metallic phase for a multicomponent alloy if $\mathrm{G}_{\mathrm{SS}}<\mathrm{G}_{\mathrm{IM}}$, where $\mathrm{G}_{\mathrm{SS}}$ and $\mathrm{G}_{\mathrm{IM}}$ denote the Gibbs free energy of the solid solution and intermetallic phase, respectively. In terms of entropy and enthalpy, this inequality can be written as $\mathrm{H}_{\text {mix }}-\mathrm{TS}_{\text {mix }}<\mathrm{H}_{\mathrm{IM}}-\mathrm{TS}_{\mathrm{IM}}$, [9]

Where $\mathrm{H}$ and $\mathrm{S}$ denote the enthalpy and entropy, respectively, and the subscript IM denotes intermetallics. As $\mathrm{S}_{\mathrm{IM}} \sim 0$, according to this, the inequality can be simplified to $\mathrm{TS}_{\mathrm{mix}}=\mathrm{H}_{\mathrm{SS}}-\mathrm{H}_{\mathrm{IM}}$. According to previous studies, Hmix is usually approximated using the Miedema formula, Similarly, if the $H_{I M}$ of a multicomponent alloy can be approximated from the formation enthalpy of the individual binaries, we may further simplify the criterion $\mathrm{TS}_{\text {mix }}=\mathrm{H}_{\mathrm{SS}}-\mathrm{H}_{\mathrm{IM}}$.

Based on a previous work ${ }^{[11]}$, it has been shown that to form a solid solution, there should be a small atomic radii difference $(\boldsymbol{\delta}<6.5 \%$ ), near-zero (absolute) value of $\Delta_{\text {mix }} H_{\mathrm{m}}$ (-15 to $\left.5 \mathrm{~kJ} / \mathrm{mol}\right)$ and a high entropy of mixing $\left(\Delta_{\text {mix }} S_{\mathrm{m}}\right)(12-17.5$ $\mathrm{J} / \mathrm{Kmol})$.

$T \Delta_{\text {mix }} S_{\mathrm{m}}$ may compete with $\Delta_{\text {mix }} H_{\mathrm{m}}$ at high temperature, following the expression: $\Delta_{m i x} G \mathrm{~m}=\Delta_{m i x} H_{\mathrm{m}^{-}} T \Delta_{m i x} S_{\mathrm{m}} . \Delta_{m i x} H_{\mathrm{m}}$ may thus be regarded as driving force for solid solution formation and $T \Delta_{m i x} S_{\mathrm{m}}$ as the resistance to formation of solid solution ${ }^{[12]}$. It has been reported in literature that $\Omega>1$ and $\boldsymbol{\delta}<6.6 \%$, simple solid solutions form. Besides, with high VEC, FCC is reported to be highly stable and BCC is found to be stable at low VEC.

\section{Major properties of $\mathrm{HEAs}^{[10]}$}

Severe Lattice Distortion: This effect arises due to the fact that there exist multicomponent matrices in HEAs mainly due to atomic size difference in individual elements. This leads to severe lattice distortion and not only, affects various properties like strength, hardness e.t.c but also reduces the thermal effect 
on properties. By the term "lattice" in HEAs, the average lattice arising due to formation of different solid solutions, is being referred to. Strength is expected to increase due to increased solid solution strengthening occurring due to severe lattice distortion. Besides lattice distortion, the other factors contributing to the same are: different bonding energies, and difference in crystal structure(arising due to different efficiency of packing). This effect is enhanced when relative difference in atomic radii between the individual elements is very high and viceversa.

Sluggish Diffusion Effect: HEAs contain mainly random solid solution, intermetallic compounds and/or ordered solid solution. Thus, atomic diffusion in whole-solute matrix is very different than that in a matrix of a conventional alloy. A vacancy in a whole-solute matrix is surrounded and competed by different element atoms during diffusion, due to large fluctuation in lattice potential energy(LPE) between lattice sites. The abundant low LPE sites may serve as traps and hinder atomic diffusion. This causes Sluggish Diffusion Effect.

\section{The concept of Compositional Complexity in alloys}

Many literatures ${ }^{[5][6]}$ report that Compositionally Complex Alloys are those which were initially termed as HEAs, in 2004. According to the earliest definition, proposed by Yeh, at al. ${ }^{[2]}$ HEAs must have at least 5 elements in equiatomic or near-equiatomic $(\sim 5-35 \%)$ proportions, in order to maximise the molar entropy of mixing $\left(\Delta_{m i x} S_{\mathrm{m}}\right)$. But, at present, this definition of HEAs is found to be inconsistent with the basic notion of maximisation in molar entropy of mixing $\left(\Delta_{m i x} S_{\mathrm{m}}\right)$ and thus, the new term "Compositionally Complex alloys" has emerged. For instance, a 4-component equiatomic alloy, AlTiVCr was reported $^{[15]}$ to possess a single phase $\mathrm{B} 2$ (ordered BCC solid solution) at room temperature, in its as-cast microstructure, such that the molar enthalpy of formation of B2 phase $\left(\sim \Delta_{f} H_{\mathrm{m}}=-9.30 \mathrm{~kJ} / \mathrm{mol}\right)$ was revealed to be lesser than that for disordered BCC phase $\left(\sim \Delta_{f} H_{\mathrm{m}}=-1.25 \mathrm{~kJ} / \mathrm{mol}\right)$ at low temperature, from the same study. Besides, it was also found, from the same study, that, the values of $\Delta_{m i x} S_{\mathrm{m}}$ and $\Delta_{m i x} H_{\mathrm{m}}$ were in agreement with intermetallic compound formation whereas only the values of $\boldsymbol{\delta}$ and $\Omega$ showed that solid solution should form. However, from the characterisations, carried out later, it was observed that single phase B2, in this alloy, was found to be stable over disordered BCC, over a wide range of temperature. Thus, the established fact that AlTiVCr forms a single phase B2 over a wide range of temperature, is similar in analogy with respect to the 5-component equiatomic Cantor alloy ${ }^{[3]}, \mathrm{CoCrFeMnNi}$, forming a singlephase FCC at room temperature and defies the earliest definition of HEAs where at least 5 components are required in equiatomic or near-equiatomic proportions 
are required to exhibit the "High-entropy" effect ${ }^{[2]}$ and form only single phase/ multi phase solid solutions or single/multi-phase solid solutions along with intermetallic compounds.

\section{Summary and Outlook}

The advent of HEAs, composed of multiple principle elements unlike conventional alloys with mostly, one and very rarely, two base elements, has fully revolutionised the concept of alloy designing and also led to a lot of modern theories, methods and even, models of alloy designing which not only apply to HEAs but also to conventional alloys. However, these theories and models(especially, those for atomistic movement governing phase formation ${ }^{[16]}$ ) need to be investigated, further, and although, there has been a lot of work on mechanical, physical as well as chemical properties of HEAs, but a lot remains to be done, primarily because of two reasons: firstly, the formation of different phases in HEAs, needs to be explored, in depth, although a certain amount of work has been done on the phase stability of HEAs and secondly, a very large number of possible combinations in HEAs. The latter may prove to be useful but the major challenge lies in identifying the useful alloy composition for a particular application, through trial and error method, which turns out to be a highly cumbersome and even, a non-economic task ${ }^{[5]}$. Besides, the new concept of "Compositional complexity" also needs to be explored, in depth, in order to draw a proper conclusion as to whether the old concept of "High-entropy alloys" may be completely, replaced by the new definition of "Compositionally Complex alloys" and if exceptions exist, then which compositions are those? and why are they exceptional? However, in spite of all challenges, HEAs are found to possess excellent novel properties such as high specific strength, superconductivity, superparamagnetism, excellent mechanical properties at elevated temperature, excellent ductility and fracture toughness at cryogenic temperature ${ }^{[4]}$. For instance, HEAs, as light as Al, but stronger than Metallic glasses, have been investigated to be useful for transport and energy sectors where "lightweight" materials have a huge demand due to reduction in fuel consumption and thus, minimising the energy requirements ${ }^{[16-20]}$. Refractory HEAs find application in high-temperature applications like gas turbines, rocket nozzles, nuclear construction e.t.c whereas, Lightweight refractory HEAs find applications in aerospace applications like re-entry nosecones. In view of the promising applications, HEAs, with promising properties, may be designed with further advancements, thereby promoting their usage in industries, which is limited, even till today.

\section{Acknowledgement}


The author is grateful to all the faculty members and Ph.D scholars from the department of Metallurgical and Materials Engineering, NIT Durgapur, for their extreme support and dedication to carry out fruitful discussions in the present regard.

\section{References}

[1] R.E. Hummel, Understanding Materials Science, second ed., Springer-Verlag, 2004.

[2] J.W. Yeh, et al. Adv. Eng. Mater. 6 (5) (2004) 299.

[3] B. Cantor, et al. Mater. Sci. Eng. A 375-377 (2004) 213.

[4] Y.F. Ye, et al. Scripta Mater. 104 (2015) 53.

[5] Y. Zhang, et al. Progr. Mater. Sci. 61 (2014) 1.

[6] B. Gludovatz, et al. Science 345 (6201) (2014) 1153.

[7] M.A. Hemphill, et al. Acta Mater. 60 (16) (2012) 5723.

[8] S.Q. Xia, et al. JOM (2015) 1.

[9] Y. Zhang, et al. Adv. Eng. Mater. 10 (6) (2008) 534.

[10] R.A. Swalin, Thermodynamics of Solids, Wiley, 1962.

[11] K. Guan- $\mathrm{Yu}$, et al., FCC and BCC equivalents in as-cast solid solutions of $\mathrm{Al}[\mathrm{x}] \mathrm{Co}[\mathrm{y}] \mathrm{Cr}[\mathrm{z}] \mathrm{Cu}[0.5] \mathrm{Fe}[\mathrm{v}] \mathrm{Ni}[\mathrm{w}]$ high-entropy alloys, Cachan, France, 2006.p. 669.

[12] H.Y. Ding, K.F. Yao, J. Non-Crystal. Solids 364 (1) (2013) 9.

[13] J.Y. He, et al. Acta Mater. 62 (2014) 105.

[14] O.N. Senkov, et al. Nat Commun. (2015) 6.

[15] Y. Qiu, et al. Acta Mater. 123 (2017) 115-124.

[16]. Y.F. Ye, et al. Materials Today 19 (2016) 349-362.

[17] M. Saha, " $\gamma$-TiAl alloy: revisiting tensile creep deformation behaviour and creep life at $832{ }^{\circ} \mathrm{C}$," https://doi.org/10.1080/2374068X.2021.1949175, 2021, doi: 10.1080/2374068X.2021.1949175.

[18] M. Saha, "A brief discussion on the tensile creep deformation behaviour of wrought single-phase $\gamma$-TiAl," Materials Today: Proceedings, Jan. 2021, doi: 10.1016/j.matpr.2020.11.189.

[19] M. Saha, "Understanding the role of $\mathrm{Al} 2 \mathrm{O} 3$ formed during isothermal oxidation in a dual phase AlCoCrFeNi2.1 Eutectic High-Entropy Alloy," Journal of Materials NanoScience, vol. 7, no. 2, pp. 68-72, Nov. 2020, [Online]. 
Available: http://thesciencein.org/journal/index.php/jmns/article/view/119

[20] M. Saha and M. Mallik, "Additive manufacturing of ceramics and cermets: present status and future perspectives," Sädhanā 2021 46:3, vol. 46, no. 3, pp. 135, Aug. 2021, doi: 10.1007/S12046-021-01685-2. 